\title{
ATTD 2019 Abstract Author Index
}

A Pinto, V., 154

A Q Oliveira, J., 154

A. Vigersky, R., 36

Aanstoot, H.J., 279

Abdel-Latif, G., 37

Aberer, F., 151, 163, 205

Abreu Abreu Padin, C., 165

Abreu Lomba, A., 309, 385

Abreu, C., 148, 156

Adamov, A., 174

Adolfsson, P., 294

Adolph, K., 214, 271

Agarwal, G., 261

Aghili, R., 138

Agnello, L., 136

Aguiar Medeiros, M., 224

Aguirre, M., 278

Agustin, B., 81

Ahmed, A., 134, 351

Ahmetov, I., 340

Ajith, J., 258

Ajjan, R., 249

Ajjan, T., 249

Ajsic, A., 152

Åkesson, K., 130

Al Khalaf, F., 366, 379

Al Sabbah, H., 125

Al-Kardsheh, A., 296

Al-Yafi, K., 377

Alasbali, M., 334

Albanese-O’Neill, A., 143

Albuhayjan, R., 334

Alcantara-Aragon, V., 320

Alcarria, E., 156

Aldea, A., 166

Alhakami, A., 391

Ali, W., 387

Aliste, M., 281

Alketbi, M., 125

alkhalifah, R., 391

Allender, S., 323

Alluis, B., 73, 76, 291

Almeda-Valdes, P., 269

Almutairi, F., 334

AlMutairi, S., 334

Alonso, N., 280

Alqahtani, T., 334

Alshaigy, B., 166

Alves, C., 346, 347

Åm, M.K., 107, 110, 209

Amato, A., 314

Ambrosch, G.C., 163
Ametov Sergeevich, A., 101

Amorim Wildes, W., 350

Ampudia-Blasco Javier, F., 290

Anastassiadis, E., 291

Anderson, E., 231

Anderson, L., 117

Andersson, Y., 285

Andrade, R., 333

Andreeva, L., 146

Aneji, C., 128

Anez-Zabala, C., 264

Anjana, R.M., 323

Antillon-Ferreira, C.A., 269

Antinori-Lent, K., 99

Antypas, K., 339

Aradóttir, T.B., 158

Arens, J.H., 318

Argimatova Shevketovna, G., 101

Arnaldi, C., 246

Arora, M., 287

Arrieta, A., 186

Arroyo Díez, F.J., 245

Arroyo-Díez, F.J., 361, 66

Arroyo-Díez, J., 220

Arrubla, J., 73

Årsand, E., 253, 254, 255, 339, 376

Arteiro, C., 288

Aschemeier, B., 271

Askarov, M., 305

Assaloni, R., 228

Astin Astin, K., 249

Åstrand, M., 303

Atkins, M., 104

Atlas, G., 285

Attia, H., 37

Augustin, T., 163

Austin, M., 173

Avari, P., 41, 166, 244

Avari, P.E., 192

Avbelj Stefanija, M., 8

Ayyagari, U., 134, 201

Azadi, N., 138

Azam Iqbal, S., 351

Azmi, L., 307

Babikian, S., 82

Bach, L., 67, 230

Bachmann, S., 337

Badarudeen, S., 135

Bagdemir, E., 207

Bailey, C., 1

Bailey, R., 143
Bailey, T., 56, 88

Bailey, T.S., 251

Baillot-Rudoni, S., 283

Bajpai, S., 344

Bakhtani, P., 162, 164

Balachandran, K., 257

Baladassano, S., 314

Balan, A., 181

Balanova, Y., 79

Balantac, Z., 344

Bally, L., 40, 120, 121, 357

Balogh, E., 344

Baltazar, R., 288

bandehpour, M., 388

Banks, P.L., 315

Baptista, C., 39, 141, 142

Barahona, M.J., 280

Baranov, V., 335

Barbed, S., 204

Barbitoff, Y., 335

Barnard D., K., 117

Barnard, K., 17, 21

Barnes, S., 285

Barone, M., 348, 350

Barrasso, M., 234

Barrio-Castellanos, R., 66

Barrio, R., 220

Barsukov, I., 276, 277, 368

Bashnina, E., 335

Basu, A., 116, 226

Basu, R., 116, 226

Batel Marques, F., 346, 347

Battelino, T., 8

Baumstark, A., 237

Beato Víbora, P.I., 245

Beato-Víbora, P., 66, 210, 220, 361

Beck-Nielsen, H., 328

Beck, P., 152

Beck, R., 4, 13

Bedoya Joaqui, V., 309, 385

Beikmohammadi, L., 388

Bellia, C., 10, 136

Bém, R., 343

Bengtsson, H., 158

Benhamou, P.Y., 111

Bergenstal, R., 74

Berget, C., 45, 48, 197

Bergman, B., 75

Berseneva, O., 335

Bertachi, A., 159, 160

Bertaina, S., 342

Bezerra Parente, E., 224 
Bezerra, V., 350

Bhattacharyya, S., 381, 381

Biagi, L., 159, 160

Biasoni, C., 358

Bidet, S., 171

Biester, S., 214, 271

Biester, T., 214, 242, 271, 302

Bindra, N., 98

Biradar, S., 181

Birnbaumer, P., 151, 205

Bjornstad, P., 267

Blaabjerg, L., 328

Blanc, R., 111

Blixgård, H., 254, 255, 376

Bloethner, S., 318, 324

Boda, H.R., 134

Bode, B., 56

Bode, B.W., 73

Böhm, L., 174

Boiroux, D., 158, 359

Bolli, G.B., 93

Bolotko, Y., 153

Bondia, J., 113, 159

Bonetti, L., 71

Bonfadini, S., 60

Bonfanti, R., 246

Bongiorno, C., 234

Bonura, C., 246

Boochandran, T., 201

Bösch, P.C., 209

Bosi, E., 69, 72, 234

Bosnyak, Z., 93

Boss, A., 310

Bottari, S., 86

Bouaoun, R., 100

Boughaleb, H., 313

Boughton, C., 120, 121

Bouillet, B., 283

Boulton, D., 302

Boulton, D.W., 303

Boyle, J., 345

Bracken, R.M., 151, 205

Bradway, M., 254, 255, 339, 376

Brændholt Olsen, M., 80

Brain, J., 94

Bramlage, P., 95, 293

Bratina, N., 8

Braune, K., 38, 112

Brazg, R., 56, 88

Bredie, S.J., 62, 256

Breton, M., 44, 119, 170, 185, 341, 342

Breton, M.D., 310

Brewer, J., 296

Brindisi, M.C., 283

Briner, A., 40

Broj, C., 99

Brovko, M., 330, 389

Bruce, S., 74

Brunato, B., 228

Buckingham, B., 48, 58, 222, 336

Bueber-Dickin, V., 319

Burcez, C.T., 100

Burt, M., 67, 230

Buse, J., 94
Buse, J.B., 315

Busse, D., 302

Büsser, A., 174

Bustamante-Martinez, J.F., 269

Butler, A., 217

C R Cimini, C., 154

Cai, K., 231

Caithaml, V., 265

Cakmak, R., 207

Caldeira, M., 141, 142

Caleca, N., 171

Cali, A.M.G., 93

Camalon, T., 171

Camerlingo, N., 232

Campbell, A., 176, 184

Campbell, F., 186, 249

Campbell, J., 371, 379

Campo Rivera, N., 309

Cappon, G., 42, 232

Cardoso, L., 39

Caretto, A., 69, 234

Cariou, B., 315

Carlsen, S.M., 107, 110, 209

Carmo Redondo, M., 288

Carrilho, F., 39, 141, 142

Carvajal Ortiz, R., 309, 385

Carvalho, D., 288

Casanova Valderrama, M.E., 309, 385

Casellato, F., 375

Cases, J., 296

Castañeda, J., 72

Casteels, K., 70

Castellà, M., 208

Castenada, J., 186

Castiglioni Teresa, M., 69

Castillo-Galindo, C., 269

Castle, J., 3, 65, 108, 172

cavalli, C., 71

Ceballos-Macías, J.J., 269

Ceceña-Gonzalez, M.P., 269

Cellai, C., 69

Chan, C., 267

Chan, J.C., 59

Chan, Y.P., 76

Chang, A., 56

Charleer, S., 55, 70

Charlesworth, T., 233

Charpentier, G., 111

Charvet, R., 76

Chaudhary, N.K., 372

Chawla, R., 162, 164

Chen, H., 114, 188

Chen, J., 182

Chen, X., 187, 193

Cheng, A., 93

Chhabra, M., 98

Chico Ballesteros, A.I., 260

Ching, T.H., 224

Choudhary, P., 72, 186

Chow, E., 59

Chowdhury, F., 215

Christensen, M.B., 43, 300, 312

Christiansen, M., 56, 88, 97
Christiansen, S.C., 107, 110, 209

Christodoulidis, S., 105, 263

Christopher, W., 247

Chrzanowska, J., 353

Chua, C., 355

Chua, S., 68

Chugunova, E., 146

Chujo, D., 212

Ciaccio, M., 10, 136

Cimino, E., 60

Cinar, A., 113

Citro, G., 228

Clarke, P., 67, 230

Clay, M., 222

Clay, S.M., 58

Cleal, B., 38, 112

Cobelli, C., 116, 190, 226

Cohen, N., 67, 194, 230

Cohen, O., 72

Coll, A., 120, 121

Colman, P., 67, 230

Colman, P.G., 194

Colmegna, P., 341

Combalia, N., 221

Conget, I., 159, 208, 220

Contreras, I., 160

Corbett, J., 170

Cordero, T., 49, 187, 193

Correale, L., 60

Correia, J., 73, 96

Cortes, M., 350

Cosma, C., 136

Costa, A., 288

Covington, K., 233

Cracowski, J.L., 86

Cruz-Bravo, M., 156, 165

Cruz, V., 240, 241

Cukier, P., 250

Cummins Cummins, M., 97

Cutruzzolà, A., 228

Cuttriss, N., 264

Cyganek, K., 289

d'Annunzio, G., 246

Da Silva, J., 72

Dachis, J., 327

Daems, C., 313

Daghero, A., 225

Dalla Man, C., 116, 157, 226

Dandona, P., 77, 306

Danielyan, A., 304

Danielyan, H., 304

Daniiels, J., 155

Danne, T., 51, 95, 214, 271, 293, 302, 315

Dar, S., 202

Darenskaya, M., 102, 146, 298, 299

Das, K., 201

Das, S., 134, 201

Dash, K., 134

Dassau, E., 179

David, A., 258

Davies, J., 132

de Azevedo, D.P., 250

De Battista, H., 198 
De Block, C., 55, 70

de Bock, M., 67

de Galan, B.E., 62, 256, 297

De Jong, C., 279

de la Brosse, L., 171

De La Garza-Hernandez, N.E., 269

De Leiva, A., 320

De Marziani, G., 225

De Portu, S., 178, 186, 72, 236

De Ridder, F., 70

de Souza, A.B.C., 250

De Valk, H., 72

De Vries, M., 279

Debock, M., 230

Dee, S., 321, 325

Deev, A., 79

Dekker, P., 279

Del Favero, S., 115, 190, 232

Del Miglio, R., 71

Delgado, M., 278

Demina, A., 276, 277

den Brinker, M., 70

Denhaerynck, K., 337

Denham, D., 56

Derdzinski, M., 52, 54, 87

DeSalvo, D., 57, 243

Devisme, C., 93

Devisscher, C., 103

Dharamalingam, M., 181

Dhatariya, K., 239

Di Camillo, B., 50, 203

Dianaty, A., 183

Didangelos, T., 168, 169

Dietz, P., 151, 205

Diez, J.L., 113

DiMeglio, L., 243

Dinccag, N., 207

Diouri, O., 171

Dirnena-Fusini, I., 107, 110

Ditkovskaya, L., 335

Dodier, R., 63, 65, 172

Doksanský, M., 265

Donner, B., 337

Donsa, K., 163

Doron, M., 111

Douvas, A., 341

Dovc, K., 32

Dovile, V., 247

Doyle III, F.J., 179

Doyle, G., 38, 112

Doyle, M., 296

Dozio, N., 69, 234

Dreval, A., 262, 276, 277, 368

Dreval, O., 277

Driscoll, K.A., 45

DuBois, J., 144, 145

Dubský, M., 343

Duce, D., 166

Duke, S.A., 248

Dunn, T., 51, 89, 213, 233

Duracher, D., 96

Dutt-Ballerstadt, R., 231

Dutta, S., 189

Duun Henriksen, J., 328
Dwarakanath, C., 201

Dyal, N., 261

Dymond, E., 249

Ebrahim Valojerdi, A., 138

Eckstein, M.L., 151, 205

Edelman, S., 306

Ehrmann, D., 29

Eichorst, B., 173, 378

Eirik, A., 316

Eisler, G., 295

Ekholm, E., 303

El Fathi, A., 167

El Youssef, J., 65, 172

Elander, M., 108

Elbert, A., 225

Ellingsen, R., 209

Elmosalami, D., 37

Elvira, A., 165

Ermakova, A., 354

Ernst, G., 229

Erukainure, O., 384

Escobedo-Ortiz, A.R., 269

Esteves-Sanchez, P., 269

Esteves, C., 288

Evans, M., 120, 121

Fabris, C., 44, 342

Facchinetti, A., 42, 50, 232

Faccioli, S., 115

Faradji, R., 269

Farías, M., 275

Farina, M., 355

Fatehi, F., 317

Faulds, E., 380

Fawziya, A.K., 371

Fedyakov, M., 335

Feig, D., 236

Feinberg, J., 179

Fejfarová, V., 343

Feldhahn, L., 242

Felgueiras, P., 148

Fendler, W., 219

Feniger, E., 202

Fernández-Balsells, M., 41, 166

Fernández-Pombo, A., 218

Fernández-Real, J., 41

Fernández-Real, J.M., 166

Ferraro, O., 60

Ferreira-Hermosillo, A., 269

Ferrer, M., 204

Figueroa-Andrade, M.H., 269

Filipp, S., 264

Fir, M., 231

Fisher, H., 371, 379

Fisher, W., 251

Flekač, M., 329

Floether, F., 174

Florenza, G., 48

Forlenza, G., 58, 197, 222, 267

Forlenza, G.P., 45

Forsander, G., 292

Fortier, C., 76

Foster, N., 143
Fouad, W., 37

Fougner, A.L., 107, 110, 209

Fraga, D., 355

Franc, S., 111

Freckmann, G., 227, 237, 238, 286

Frias, J., 93

Frier, B., 75

Fritzen, K., 338

Frongia, A.P., 246

Fukui, T., 133

Fuqua, L., 173

Furtado, P., 141, 142

Gabarron, E., 316

Gaber, A.O., 355

Gaede, P., 43

Gaipov, A., 305

Galastri, L., 348

Galderisi, A., 175

Galhaud, J.P., 149

Gallego Gamero, F., 245

Gallego-Gamero, F., 210

Gamarra, E., 342

Gambino, C.M., 136

Gandhi, S., 365

Gantenbein, C., 337

García Jaramillo, M., 240, 241

García Lobato, C., 245

Garcia-Chamon, R.B., 221

Garcia-Garcia, R., 196

García-López, J.M., 218

Garcia-Seco, F., 278

Garcia-Seco, J.A., 278

Garcia-Tirado, J., 341

Gardner, D.S.L., 36

Garelli, F., 198

Garg, S., 14, 315

Garvey, K., 91

Garza, K., 117

Gaudier, M., 73, 96, 291

gautam, A., 307

Gautier, T., 310

Geelhoed-Duijvestijn, P., 247

Geissler, A., 76

Genua Trullos, I., 260

Georgiou, P., 20, 106, 155, 182, 192

Gernay, M.M., 223

Gianello, P., 100

Giannoulaki, P., 168, 169

Gies, I., 103

Gil Poch, E., 245

Gil-Poch, E., 66, 220

Gillar, D., 265

Gillard, P., 55, 70

Gillon-Keren, M., 6

Giménez-Álvarez, M., 66

Giménez, M., 159, 208, 220

Giordanengo, A., 255, 376

Girardot, S., 122

Girelli, A., 60

Giri, R., 46

Glotov, A., 335

Glotov, O., 335

Gnusina, S., 299 
Godtliebsen, F., 367

Goebel, B., 157

Gomez-Cruz, J.R., 269

Gomez-Peralta, F., 156, 165

Gomez-Rodriguez sara, S., 165

Gomez-Rodriguez, S., 156

Gomez, A.M., 240, 241

Gonnet, N., 86

Gontard, P., 149

González Blanco, C., 260

Gonzalez-Galvez, G., 269

Gonzalez, C., 320

Gonzalez, M., 218

Gooley, J., 46

Gorodner, V., 358

Gotfredsen, A., 43

Graham, C., 206

Grant, M., 74, 94

Grassi, B., 275, 281, 284

Grattoni, A., 355

Grebenkina, L., 102, 298, 299

Greenfield Greenfield, M., 82

Greening, J., 104

Greenwood, D.A., 173

Griffith., G., 290

Grineva, E., 153

Griva, F., 282

Grosman, B., 176, 183, 184

Grunberger, G., 354

Gubar, E., 349

Gubkina, V., 262

Güemes González, A., 106

Guibat-Demont, A., 348

Gul, N., 207

Gunesekera, D., 382

Guo, X., 68

Gupta- PhD, P., 217

Gupta, A., 252

Gurfinkel Ilyich, Y., 101

Hadad, Y., 150

Haidar, A., 167

Haller, M., 264

Hamddan Lachkar, M., 103

Hamed, M., 37

Hameed, S., 248

Hampton, J., 321, 325

Hamren, B., 303

Hána, K., 265

Hanberger, L., 130

Hanes, S., 58, 222

Hansart, A., 103

Hansen, L., 77

Hardy, S., 122

Hardy, T., 68

Harrington, K., 243

Harrison, R., 166

Hartnell, S., 120, 121

Hatzitolios, A., 168, 169

Hauck, B., 38, 112

Haug, C., 227, 237, 238, 286

Hauth, W., 318

Hayashi, T., 133

Hayter, G., 51, 89, 233
He, X., 344

Heise, T., 2, 73, 80, 291

Helm, N., 294

Henao, D., 240, 241

Hendrieckx, C., 67, 230

Herbrand, T., 291

Herchcovici, S., 109

Hererro, P., 155

Hermann, J., 57, 242, 293

Hermanns, N., 29

Hernando, M.E., 320

Hero, M., 131

Herrero Viñas, P., 192

Herrero, P., 20, 41, 106, 166, 182

Hershcovitz, Y., 202

Herz, A.M., 43

Herzig, D., 40, 121

Heschel, M., 336

Hess, G.M., 95

Hewitt, J., 285

Heyman, M., 327

Hilliard, M., 243

Hinzmann, R., 174

Hirano, T., 133

Hiromura, M., 133

Hirota, Y., 53

Hirsch, A., 327

Hirsch, I., 5

Hjelme, D.R., 209

Hochfellner, D., 152

Hoff, A.A.O., 250

Hofmann, P., 151, 205

Højlund, K., 328

Holl, R., 57, 95, 242, 293

Holler, P., 205

Holmes-Walker, D., 67, 230

Holubová, A., 265

Hommel, E., 43

Hood K., K., 117

Hood, K., 18, 81, 222

Hood, K.K., 58

Horsburgh, J., 67

Horsburgh, J.C., 194

Hosny Awad, M., 71

Hout, T., 268

Hövelmann, U., 68, 75, 80, 96

Hovorka, R., 120, 121

Hsu, L., 336

$\mathrm{Hu}, \mathrm{H} ., 264$

Huang Huang, S., 187

Huang, J.S., 331

Huddleston, B., 327

Huhn, F., 229

Humayun Nuzhat, K., 351

Hundal, R., 296

Hunt, P., 356

Huschto, T., 174

Hussain, K., 366, 371, 379

Hwang, J.Y., 331

Hynes, S., 51

Iacolino, G., 136

Iglesias, M.L., 225

Imaeva, A., 79
Imitola, A., 240, 241

Iniesta, J., 320

Iqbal, R., 351

Irace, C., 228

Isitt, J., 206

Islam, M., 384

Islam, M.S., 301

Islam, S., 311, 386

Islas-Ortega, L., 269

Ivashchenko, T., 335

Jack, M., 248

Jackson, C., 143

Jacobs, P., 63, 65, 172

Jacobs, S., 70

Jacoby, J., 378

Jaganmohan, B., 134

Jallon, P., 111

Jana, B., 369

Janani, L., 138

Janež, A., 90

Jangam, S., 89, 233

Jankovic, M.V., 195, 352, 357

Janocha, A., 353

Jaramillo, A., 273

Jelenina, L., 335

Jena, S., 323

Jendle, J., 178, 306

Jendrike, N., 227

Jenkins, A., 46, 67, 83, 194, 230

Jensen, M.L., 158

Jian, Z., 137

Jiletcovici, A., 344

Jimenez, A., 52, 54, 87

Jindal, H.A., 92

Jiráňová, P., 47, 191

Jirkovská, A., 343

Jirkovska, J., 373

Johnson, J., 326

Johnson, K., 308

Johnsrud, M., 308

Jone, H., 176, 183, 184

Jones, H., 46, 83

Jones, H.M., 194

Jones, R.S., 326

Jones, T., 67, 230

Jördening, M., 229

Jørgensen, J.B., 359

Joseph, J., 295

Joshi, S., 162, 164

Joshi, S.R., 134

Jothydev, S., 135, 258

Judith, C., 366

Jugnee, N., 41, 166, 244

Juhl, C., 328

Junaidi, M.K., 97

Jussila, R., 130

Kačer, P., 329

Kajio, H., 212

Kallis, A., 282

Kamecke, U., 286

Kamel, S., 37

Kan, W.C., 331 
Kanapka, L., 61, 243

Kapitza, C., 68

Kapustina, A., 79

Kar, P., 325

Kara, F., 275

Karia, D., 287

Karibekov, T., 305

Karlafti, E., 168, 169

Karlsson, B.G., 292

Karnoukhova, O., 146

Kasamanian, M., 150

Kašpar, J., 265

Katherine, V., 147

Kato, K., 53

Kaufman R, F., 184

Kaufman, F., 49, 72, 176, 187, 193

Kaufman, F.R., 183

Kaufman, N., 78

Kaunisto, K., 131

Kawashima, S., 53

Kaye, J., 67, 230

Kazda, C., 68

Kazemi, B., 388

Kearney, R.E., 167

Kechadi, T., 38, 112

Keech, A., 67, 230

Keller, J., 336

Kendall, D., 74, 94

Kennon, B., 338

Kepaptsoglou, O., 282

Kern, W., 150

Kesavadev, J., 135, 258

Kesserwan, C., 118

Khalf, A., 295

Khamnueva, L., 146

Khan Habib, A., 351

Khasanova, K., 340

Khramova, E., 124

Kiiveri, S., 130

Kim, N., 305

Kiseleva, T., 340

Klabunde, T., 157

Klaff, L., 56, 88

Klein, D., 94

Klein, O., 73, 291

Klementová, M., 343

Klinkenbijl, B., 206

Klueh, U., 118, 360

Ko, W., 38, 112

Kobalyan, A., 100

Koehler, G., 151, 205

Koeneman, M., 62, 256

Kohata, Y., 133

Kojzar, H., 151, 205

Kolesnikov, S., 102, 146, 298, 299

Kolesnikova, L., 102, 146, 298, 299

Kollan, A., 132

Koltakova, A., 349

Kolukula, V.K., 134, 201

König, H., 174

Kontoninas, Z., 168, 169

Koorbanally, N., 311, 386

Kopanz, J., 163

Kordonori, O., 271
Kordonouri, O., 214

Korotaeva, T., 349

Korsakova, I., 349

Kos, P., 373

Koshmeleva, M., 124

Kottmann, T., 214

Kotzakioulafi, E., 168, 169

Kovaleva, Y., 262

Kovatchev, B., 19, 31

Kovatchev, B.P., 310

Kowalyk Kowalyk, S., 296

Kownacka, A., 247

Kozak, M., 191

Kozina, L., 305

Kozlov, V., 330, 389

Kraemer, K., 175

Krcma, M., 274

Kress, S., 293

Kreutzer, D., 118, 360

Krishnakumar, A., 162, 164

Krishnamoorthy, P., 167

Krishnamurthy, B., 83

Krishnan, G., 135, 258

Krivánek, R., 59

Kriváneková, L., 59

Křižová, M., 343

Kronshage, B., 80

Kučera, T., 265

Kuchieva, A., 330, 389

Kulzer, B., 29

Kumareswaran, K., 67, 230

Kuroda, A., 53

Kurtz, N., 176, 184

Kushner, J.A., 315

Kushner, T., 185, 189

Kusova, H., 274

Kuvshinov, R., 149

L Ribeiro, A., 154

La Gerche, A., 46

Lablanche, S., 72

Laffel, L., 61, 117, 243

Lai, M., 345

Laine, T., 131

Lal, R., 336

Lalic, N., 26

Lam, D., 56

Lamers, D., 80, 96

Lane, K., 239

Lang, J., 89, 233

Lange, K., 229

Lange, S., 197

Langkilde, A.M., 77, 306

Lanning, M., 81

Lánská, V., 343

Lantigua, H., 139, 363

Lanzinger, S., 95

Lapatto, R., 131

Larramona, G., 204

Laryushina, Y., 319

Lason, I., 289

Laszki-Szcząchor, K., 353

Laubner, K., 293

Launonen, I., 367
Laurenti, M., 217

Laurenzi, A., 69, 234

Lázaro Martín, L., 245

Lázaro-Martín, L., 66, 210, 220

Leal, Y., 41, 166

Lebedeva, M., 330, 389

Lebowitz, J., 173

Ledeganck, K., 70

Lee, A., 285

Lee, J., 339

Lee, J.B., 179

Lee, L.L., 36

Lee, M., 46, 67, 83, 176, 183, 184, 230

Lee, M.H., 194

Lee, S., 187, 193

Lee, S.W., 49

Lee, V., 59

Legault, L., 167

Lei, Z., 137

Leichtle, A., 40

Leiwand, B., 308

Lenzi, L., 246

Leohr, J., 68

Leon, F., 240, 241

Levy, C., 56

Lewis, D., 35, 38, 112

Li, K., 155, 182

Li, O., 330, 389

Li, X.C., 355

Li, Y., 50, 203

Libiseller, A., 163

Lichtenegger, K.M., 163

Lili, C., 362

Lim, L., 248

Lin, S., 114, 188

Lingwen, Y., 137

Link, M., 227, 237

Linn, A., 345

Lintereur, L., 176, 183, 184

Liu, C., 41, 192

Liu, K.S., 114

Liu, M., 187, 193

Liu, R., 68

Livingstone, D., 345

Lo Sasso, B., 136

Lockwood-Lee, S., 104

Loebner, S., 48

Loeum, C., 295

Loginova, E., 349

Loke, Y., 239

Lombardo, F., 246

Lonero, A., 246

Longato, E., 50, 203

López Argudo, A., 260

López, B., 166

Lotito, M.L., 355

Lou, G.M., 204

Louzado Andrade, J., 350

Lu, J., 137

Lu, Y., 105, 263

Lüddeke, H.J., 84

Lueddeke, H., 85

Lugo, S., 140, 147, 363

Lui, C., 166 
Luisa, B., 39

Luk, A.O., 59

Lumb, A., 132

Lunder, M., 90

Lupianez-Barbero, A., 320

Lv, D., 119

Lyon, A., 144, 145

Lyon, J., 186

Lyon, M., 144, 145

Lyon, O., 144, 145

Lysy, P., 70, 313

Ma, Y., 94

Maahs, D., 57, 264

MacIsaac, R., 230, 46, 67, 83, 194

Mader, J.K., 152, 163

Madero-Fernández del Castillo, M.A., 269

Madondo, K., 50, 203

Maffeis, C., 246

Magis-Rodriguez, C., 269

Magisson, J., 100

Mahmoudi, Z., 359

Malecki, M., 289

Malmodin, D., 292

Malone, J., 326

Mandelbaum, O., 150

Manikandan, R., 201

Marcolino, M., 154

Marigliano, M., 246

Marin Sanchez, A., 273

Marks, B., 91

Marques Naldi, M., 224

Marshall, A., 248

Martignoni, F., 121

Martín-Fernández, J.A., 159

Martín-Frías, M., 66, 220

Martin, C., 41, 166

Martinez Melgar, C., 260

Martínez Roldán, M.J., 260

Martínez-Ramos-Mendez, A., 269

Martinez, C., 320

Martinez, M.J., 320

Martins, D., 141, 142

Martins, P., 141, 142

Martyn-Nemeth, P., 235

Massa, G., 70

Massana, J., 166

Mathieu, C., 15, 55, 77, 306

Matsuhisa, M., 53

Mattishent, K., 239

Maurits, J., 247

May, H., 239

McAhren, S., 174

McAssey, K., 261

McAuley, S., 46, 67, 83, 230

McAuley, S.A., 194

McCallum, R., 67, 230

McCarthy, R., 290, 382

Mcdonald, L., 342

McGuire, D.K., 315

McMahon, C., 266

Medeiros, D., 350

Mégret, C., 291

Meiffren, G., 73, 76, 96, 291
Mende, J., 286

Mendes, D., 346, 347

Meneghetti, L., 190

Meo, S., 334

Merger, S., 293

Merino-Torres, J.F., 320

Messer H., L., 197

Messer, L., 24

Messer, L.H., 45

Meyer, Y., 76

Meyramov, G., 319

Michalak, A., 219

Michelson, A., 179

Micucci, D., 253

Miettinen, P., 131, 211

Miller, E., 354

Miller, K., 57, 61, 243

Milovanov, Y., 330

Milovanova, L., 330, 389

Milovanova, S., 330, 389

Minar, V., 161

Minshall, M., 206

Minuto, N., 246

Miracle-López, S., 269

Miranda, F., 148

Misnikova, I., 262, 368

Mistro, S., 350

Mitchell, B., 344

Mitrakou, A., 282

Moecks, J., 318, 324

Mohan, V., 323

Mohsen, A., 37

Monnier, M.P., 283

Montaner, T., 204

Montaser, E., 113

Montes-Valdespino, D., 269

Moreno-Fernandez, J., 278

Morosanu, A., 129

Morosanu, M., 129

Moscardó, V., 244, 220

Moser, F., 358

Moser, O., 151, 205

Mosquera-Lopez, C., 63, 65, 172

Mougiakakou, S., 105, 195, 263, 352, 357

Mouritzen, U., 80

Mousin, F., 122

Mouslech, Z., 177

Mozzillo, E., 246

Mueller, A., 151, 205

Mühldorfer, S., 95

Mul, D., 279

Mulè, F., 314

Mulka, A., 360

Müller, A., 59

Muñoz Lombo, J.P., 309, 385

Muñoz, O.M., 240, 241

Murata, T., 53

Murlidharan, S., 323

Muromtseva, G., 79

Murphy, H., 236

Murthy, H., 64

Mussri, D., 275, 281

Mustafa, A., 377
Mužík, J., 265

Mužný, M., 253, 254, 255

Nagaike, H., 133

Nagelhout, J., 332

Nally, J., 345

Nally, L., 58, 175, 222

Nambiar, R.S., 287

Namokonov, E., 102, 298

Naranjo, D., 81, 117

Nascimento do Ó, D., 333

Nasykhova, Y., 335

Natalucci, V., 60

Naudorf, M., 95

Navarro, L., 358

Naveenam, M., 64

Navodnik Preloznik, M., 231

Němcová, A., 343

Nery, M., 250

Neuman, V., 47

Neumann, V., 191

Neupane, S., 239

Neves, C., 288

Neves, J.S., 288

$\mathrm{Ng}$, J., 59

Nguyen, A., 308

Nichols, J., 355

Nicolaevna Kochergin, C., 350

Nicolucci, A., 338

Nieswandt, A., 214

Niewswandt, A., 271

Nikkel, C., 296

Nildibayeva, F., 319

Niño-Vargas, R.S., 269

Nobels, F., 55

Noczyńska, A., 353

Nogueira, J.P., 225

Nordhaug Myhre, J., 367

Nørgaard, K., 43, 158, 300, 312

Norlander, L., 48

Nuboer, R., 332

Nunes Salles, J.E., 224

Nunes, L.C.R., 250

Núñez-Aldudo, A., 218

Nyunt, O., 248

O Lima, M., 154

O’Donnell, S., 38

O'Halloran, S., 108

O'Neal N, D., 184

O'Neal, D., 46, 67, 83, 176, 194

O'Neal, D.N., 183

O'Donnell, S., 112

O'Neal, D., 230

Obeide, L., 358

Obermannova, B., 191

Obeyesekere, V., 46, 83, 176, 183, 184

Ochoa, M.F., 284

Ogionwo Lange, E., 294

Ohara, M., 133

Ohsugi, M., 212

Ojaniemi, M., 131

Oje, M., 345

Okada, A., 53 
Olde Bekkink, M., 62, 256

Olewuike, I., 199, 364, 383

Olewuike, M., 180

Oliveira, A.I., 288

Oliveira, M., 350

Oliveira, S., 288

Oliver, N., 20, 41, 155, 166, 192, 220, 244

Onetto, M.T., 275, 281, 284

Orois, A., 280

Oron, T., 77

Ortega-Rodrigo, E.M., 278

Osborn, C., 327

Osorio Toro, L.M., 309, 385

Osumili, B., 344

Owen, J., 259

Oyebode, O., 384

Ozel Yildiz, S., 207

Padhi, R., 181

Paez Mayorga, J., 355

Pagacz, K., 219

Pagán, J., 50, 203

Paldus, B., 46, 67, 83, 194, 230

Palisaitis, E., 167

Palmert, M., 23

Pandis, M., 152, 163

Panevina, A., 322

Paoletti, N., 114, 188

Papa, K., 177

Paquot, N., 223

Parakhina, V., 319

Paranjape, S., 356

Pardo, S., 251, 252

Pareja, R., 221

Parikh, N., 176, 183, 184

Park, C., 235

Park, H.J., 200

Parker, A.S., 52, 54, 87

Parkinson, J., 302, 303

Parks, L., 173

Patankar, L., 390

Patti, L., 69, 234

Patton, S., 16

Pazos-Couselo, M., 218

Pedersen, A., 292

peili, C., 362

Pelcl, T., 329

Peleška, J., 265

Penfornis, A., 283

Pennathur, S., 374

Perea, V., 280

Pereira, M., 288

Peretz, S.S., 150

Pérez, V., 284

Peters, A., 74

Peters, A.L., 315

Petit, J.M., 283

Petrich, W., 174

Petrovski, G., 25, 216, 272, 366, 371, 379

Petruzelkova, L., 34, 47, 191

Pettus, J., 356

Peuscher, H., 84, 85

Peyrot, M., 344
Pferschy, P., 151, 205

Philips, J.C., 223

Phillip, M., 77

Piccini, B., 246

Piccinno, E., 246

Pickova, K., 274

Pickup, J., 12

Pieber, T.R., 163

Pillai, C., 147

Pineda-Wieselberg, R., 348

Pinsker, J., 179

Place, J., 171

Plachy, L., 47, 191

Plasova, V., 47

Plebani, M., 136

Pleus, S., 227, 237, 238

Plum-Mörschel, L., 75, 291

Polák, J., 343

Polanco Preza, M.A., 269

Polev, D., 335

Polyakova, I., 335

Pompilio, F., 74, 94

Poornima, C., 134, 201

Popova, P., 153

Poręba, M., 353

Poręba, R., 353

Poulsen, N.K., 158

Prado-Carro, A., 196

Prázný, M., 329

Preblick, R., 356

Predeus, A., 335

Prem, S., 98

Prestrelski, S., 97

Previtali, N., 40

Price, D., 52, 54, 87, 326

Proietti, A., 225

Provenzano, F., 228

Provenzano, V., 228

Pruhova, S., 47, 191

Puhr, S., 52, 54, 87

Pujol Jiménez, I.M., 260

Pulkkinen, M.A., 130, 131, 211

Pustozerov, E., 153

Puthtann, B., 268

Pyle, L., 45, 267

Q V Sá, T., 154

Qiao, Y., 118

Quail, N., 345

quanya, S., 362

Quinn, L., 235

Quirós-López, C., 66

Quirós, C., 280, 220

Rabah, T., 37

Rabbone, I., 246

Radermecker, R., 223

Rahman, T., 215

Rai, P.E., 98

Raile, K., 38, 112

Raimond, L., 43

Rajan, S., 164

Rakhmankulov, V., 368

Rama Chandran, S., 36
Ramachandran, L., 135, 258

Ramarathnam, K., 161

Ramirez-Renteria, C., 269

Ramjan, K., 248

Randine, P., 253

Ranjan, A., 300, 312

Ranjani, H., 323

Ranson, A., 73, 76, 96

Rao, C.V., 307

Rapini, N., 246

Raposo, J., 333

Rashid, M., 113

Rastogi, R., 56

Rathi, V., 98

Ratnasingam, J., 36

Ravizza, S., 174

Raymond, J., 327

Rea, P., 345

Rea, R., 132

Reach, G., 30

Rebec, M., 231

Rebolledo-Ramirez, A., 269

Reddy, M., 20, 41, 155, 166, 220, 244

Reddy, S., 68

Rehn, M., 171

Reinisch-Gratzer, J., 163

Remus, K., 214, 271

Remvig, L.S.R., 328

Renard, E., 7, 171

Rentoul, D., 206

Repovž, G., 8

Resalat S., N., 172

Resalat, N., 63

Reusch, J., 27

Reutrakul, S., 235

Riaño-Galan, I., 196

Ribeiro, R., 333

Ricagno, E., 60

Rice, D., 173

Riddell, M., 22

Riera, F., 284

Rigla, M., 221

Rinehart, C., 380

Ritzel, R., 93

Riveline, J.P., 122

Rivera Davila, M., 140, 147

Riz, M., 157

Robert, A., 313

Robertson, D., 174

Robledo, M.A., 240, 241

Rodrigo-Cano, S., 320

Rodríguez-González, R., 218

Rodriguez-Sanchez, E., 269

Rodriguez, H., 143

Roem, K.L., 194

Romanova, O., 335

Romeres, D., 116

Romero Ugalde, H.M., 111

Romero-Zazueta, A., 269

Rooijackers, H.M., 297

Rosa, S., 69

Rosales, N., 198

Rosenstock, J., 93, 315

Roussel, R., 93, 382 
Rowley, E., 38, 112

Roy, A., 176, 183, 184

Roy, S., 392, 393

Rozas, P., 278

Roze, S., 178

Ruan, Y., 132

Rubin, G., 358

Rubio Almanza, M., 338

Rubio, N., 139, 147

Rusavy, Z., 274

Russell, A., 166

Ryden, L., 382

Saboo, B., 162, 164

Sai, J., 134

Sainz de la Maza-Viadero, M.E., 269

Sakane, N., 53

Salama, I., 37

Salama, S., 37

Sale, A., 236

Saleem, M., 370

Saleh, R., 37

Salgado Cifuentes, C.A., 309, 385

Salter, C., 239

Sami, S., 37

Samoilova, J., 124

Samuelsson, U., 130

Sanal, G., 258

Sánchez Márquez, P., 273

Sanchez-Garcia, A.M., 196

Sanchez-Ruiz, K.L., 269

Sanchez, J., 236

Sandig, D., 242

Sandy, J., 248

Sankaranarayanan, S., 185, 189

Sanni, O., 301, 311, 384, 386

Sarana, A., 335

Saremi, A., 310

Sarkola, T., 131

Sas, T., 279

Sassi, A., 100

Satha, S., 268

Saullo, M., 60

Sauque-Reyna, L., 269

Sawaki, H., 53

Sawani, S., 351

Sawhney, S., 315

Scaramuzza, A., 71, 246

Scavini, M., 69, 234

Schapira Wajman, D., 224

Schatz, D., 11

Scheerer, M., 302

Scheerer, M.F., 306

Scherbak, S., 335

Schiaffini, R., 246

Schiavon, M., 116, 157, 226

Schierloh, U., 242

Schleyer, T., 174

Schmidt, S., 43, 158, 300, 312

Schneidinger, B., 174

Schnell, O., 28, 338

Schøndorff, P.K., 336

Schott, R., 326

Schütz-Fuhrmann, I., 57
Scibilia, R., 38, 112

Seco, A.M., 278

Seifert, M., 353

Selim, N., 377

Semenova, N., 299

Serebryakova, E., 335

Serifovski, N., 43

Seroussi, C., 73, 96

Sethi, M., 48

Shabanova, E., 335

Shah, A., 147, 162, 164

Shah, S., 134, 201

Shahade, A., 390

Shaikh, M., 162, 164

Shaimardanova, G., 305

Shalnova, S., 79

Shankar, A., 135, 258

Sharma, R., 98

Shemyakina, N., 102, 298

Shen, T., 75

Sheng, T., 82

Shepherd, L., 356

Shepherd, M., 248

Sherif, K., 377

Sherr, J., 33, 75, 143, 175

Sheryazdanova, S., 319

Shestakova, E., 322

Shestakova, M., 322

Shin, J., 49, 72, 187, 193

Shin, Y.J., 123

Shinde Ujwala, D., 369

Shkolnikov, V., 79

shukla, I., 307

Shved, N., 335

Siddiqui, A.R., 351

Siebel, S., 175

Sieradzan, R., 354

Signorini, F., 358

Sigrist, S., 100

Sikes, K., 175

Silva, K., 350

Silwal, R., 310

Simi, H., 205

Simó-Servat, A., 280

Sims, C., 67, 83, 230

Singh, K.P., 98

Sink, J., 296

Siscovick, D., 50, 203

Sivasithamparam, K., 41

Skinner, T.C., 38, 112

Sklyanik, I., 322

Škrha jr., J., 329

Škrha, J., 329

Skupien, J., 289

Skvarca, A., 270

Slana Ozimič, A., 8

Slavich, E., 248

Slover H., R., 197

Slover, R.H., 45

Smaradottir, B., 316

Smith, K., 94

Smolka, S.A., 114, 188

Snel, T., 332

Snell-Bergeon, J., 267
Snoek, F., 344

Soares, D., 350

Sobieszczańska, M., 353

Sokal, E., 313

Sokolov, V., 302

Soliani, M., 71

Somali, M., 177

Son, K., 200

Sosale, A., 64, 162, 164

Sosale, B., 64

Soula, O., 73, 76, 96, 291

Soula, R., 73, 76

Soupal, J., 47, 191

Sourij, C., 151, 205

Sourij, H., 151, 205

Sozinova, E., 340

Sparacino, G., 42, 232

Speight, J., 67, 230

Spence, A., 296

Spence, R., 155

Stafford, D., 91

Stanga, Z., 105

Stankevich, L., 149

Stathopoulou, T., 105, 263

Stavdahl, Ø., 209

Steil, G., 175

Stella, P., 93

Stephan, C., 214

Stettler, C., 40, 120, 121

Stoyan, S., 51

Stranks, S., 67, 230

Strasma, P., 295

Strizek, A., 344

Strock, E., 378

Strumph, P., 315

Stuhr, A., 251, 252

$\mathrm{Su}$, J., 285

Subias, D., 221

Subramanian, S., 161

Suchkova, O., 101

Suico, J., 75

Sumnik, Z., 47, 191

Sun, Q., 195, 352, 357

Sundararajan, V., 46, 67, 83, 230

Suput Omladič, J., 8

Suspitsin, E., 335

Susto, G.A., 190

Sylvest Nielsen, S., 328

Szadkowska, A., 219

Sze, D., 354

T Barone, M., 154

Tabatabai, I., 48

Taboada, L., 240, 241

Tack, C.J., 297

Takahashi, N., 212

Takeishi, S., 126, 127

Takekoshi, S., 126, 127

Talita, B., 348

Tam, M.M., 285

Tamborlane, W., 175, 243

Tan D, G., 132

Tan, A.T.B., 36

Tan, S., 317 
Tanabe, A., 212

Tandon, N., 323

Tang, W., 302, 303

Tanha, K., 138

Tanrıkulu, S., 207

Tapia, J., 320

Tapia, L., 281

Tappe, A., 38, 112

Taranova, M., 330, 389

Tavera-Hernandez, M., 269

Tejedor, M., 367

Tekin, S., 207

Tenés, S., 320

Terzi, M., 190

Terzo, S., 314

Thampiraj, K., 135

Thomakos, P., 282

Thomas, A., 36

Thomas, M., 41

Thorén, F., 77

Thoren, F.A., 306

Thorsteinsson, B., 328

Tichey, E., 175

Tinti, D., 246

Tippisetty, S., 201

Tkachuk, A., 153

Tone, A., 53

Torjman, M., 295

Tossavainen, P., 131

Town, M., 48

Townsend, N., 248

Toyoda, M., 53

Toyoshima, M.T.K., 250

Tran, N., 144, 145

Trawley, S., 67, 230

Trentham, S., 249

Trier, J., 290, 382

Troub, T., 176, 184

Trupti, A.K., 134

Truskaller, T., 163

Tsuboi, H., 126, 127

Tsui, V., 59

Tuomaala, A.K., 131, 211

Turgunova, L., 319

Turker, F., 207

Turkunova, M., 335

Turmukhambetova, A., 319

Tweden, K., 56, 88

Tyler, N., 63, 65, 172

Tyrtova, L., 335

Tziomalos, K., 168, 169

Uduku, C., 155, 182

Ueki, K., 212

Ueno, K., 212

Ulbrich, S., 237, 238

Ulyanova, O., 305

Urbano, F., 280

Uribe-Wiechers, A.C., 269

Valadez-Capetillo, M., 269

Valeeva, E., 340

Valeeva, F., 340
Valenzano, M., 342

Valenzuela Montoya, J.C., 269

Valverde, M., 280

Van Aken, S., 70

Van Meijel, L., 297

Van Name, M., 243

Vanbesien, J., 70, 103

Vanderroost, J., 313

Vandoni, M., 60

Vasiloglou, M., 105

Vasiloglou, M.F., 263

Vaslamatzis, G., 282

Vassilyeva, N., 319

Vazhenina, A., 153

Veeze, H., 279

Vehí, J., 159, 160, 198

Venkataraman, S., 134

Ventrici, C., 246

Vergès, B., 283, 338

Vermeulen, B., 103

Vettoretti, M., 42, 50, 203, 232

Vezinet, J., 122

Vidrio-Velazquez, M., 269

Vigers, T., 45, 267

Vigersky, R., 150, 266

Villeneuve, E., 111

Viñals, C., 208

Viscido, G., 358

Visentin, R., 157

Vodickova, M., 274

Vogrin, S., 46, 67, 83, 230

Vogt, A., 121

Volčanšek, Š., 90

von dem Berge, T., 271

Von Oettingen, J.E., 167

Vorrink, L., 72

Vovk, A., 8

Vrátná, E., 343

W Endlich, P., 154

Wadhwani Ashish, D., 369

Wadwa, R.P., 45, 58, 222

Wagh, S., 390

Wagner, C., 293

Waite, M., 166

Wakim, A.P., 296

Waldenmaier, D., 237, 286

Wales, A.K., 348

Walker Walker, A., 264

Walker, T., 52, 54, 87

Wallace, J., 251

Wang, X., 93

Wangberg, S., 376

Wangberg, S.C., 254, 255

Ward, G., 46, 67, 83, 230

Ward, G.M., 194

Warrier, R., 135

Weber, I., 58, 222

Wehkalampi, K., 131

Wei, L., 137

Wei, Z., 137

Weil E.G., L., 117

Weinzimer, S., 9
Weiping, J., 137

Weis, N., 318, 324

Weissberg-Benchell, J., 117

Weissmann, J., 318, 324

Welsch, S., 313

Welsh, J., 52, 54, 87

Weydt, P., 183

White, M., 285

Whitman, M., 321, 325

Wilinska, M., 120, 121

Wilke, A., 326

Willaing, I., 38, 112

Wilson, L., 65, 172

Wilson, S., 248

Witek, P., 289

Woerner, S., 243

Wolfsdorf, J., 91

Wolpert, H., 326

Woodhead, H., 248

Wos, M., 41, 166

Wosková, V., 343

Wroblewska, N., 339

Wu, D., 176, 183, 184

Wyatt, S., 83

Wyne, K., 380

X Maia, J., 154

Xavier-Oliveira, L., 348

Xhema, D., 100

Xiaojing, M., 137

Xiaolong, Z., 362

$\mathrm{Xu}$, J., 77, 306

$\mathrm{Xu}, \mathrm{Y} ., 51,213$

Yabut Yabut, K., 264

Yadhav, M., 134

Yafi, M., 128, 139, 140, 147, 363, 377

Yamamoto, T., 133

Yenidünya Yalın, G., 207

Yepez-Rodríguez, A.E., 269

Yifei, M., 137

Youde, L.S., 248

Yun, J.M., 200

Yuqian, B., 137

Zabadaev, V., 330, 389

Zakrzewska, K., 338

Zammataro, L., 175

Zandonà, A., 50, 203

Zaninotto, M., 136

Zapata, Y., 275

Zeitler, P., 267

Zhang, S., 75

Zhou, F.L., 356

Zhuplatov, S., 251

Ziegler, R., 286

Zijlstra, E., 75, 96

Ziko, H., 152

Zivkovic, M., 216, 272

Zoupas, C., 282

Zubacheva, D., 330, 389

Zubkiewicz-Kucharska, A., 353

Zumsteg, U., 337 